\title{
Adenoviral delivery of $B 7-1$ (CD80) increases the immunogenicity of human ovarian and cervical carcinoma cells
}

\author{
MG Gilligan, P Knox, S Weedon, R Barton, DJ Kerr, P Searle and LS Young \\ CRC Institute for Cancer Studies, University of Birmingham, Edgbaston, Birmingham, B15 2TA, UK
}

The majority of tumour cells do not express immune costimulatory molecules and this may account for their inability to stimulate directly an antitumour $T$ cell response. Here we report on the construction of a recombinant E1/E3-deleted adenovirus encoding the human B7-1 costimulatory molecule. We explored the use of this vector for gene transfer to a number of human ovarian and cervical tumour cell lines, and to primary ovarian tumour material. Rapid and efficient gene transfer and expression was obtained in the majority of cases using a multiplicity of infection of 30 plaque forming units per cell. B7-1 expression was detectable at the cell surface within $12 \mathrm{~h}$ and was still detectable 10 days after infection. The immunogenicity of gene-modified tumour cells was tested in an allogeneic mixed lymphocyte tumour cell culture. Tumour cells expressing B7-1 were found to induce significantly higher levels of $T$ cell proliferation than tumour cells modified with a control adenovirus carrying the $\beta$ galactosidase gene. B7-1-induced T cell proliferation could be blocked by the addition of anti-B7-1 antibodies at the initiation of cocultures. These results support the rationale for use of adenovirally delivered B7-1 for genetic immunotherapy of ovarian and cervical cancer.

Keywords: B7.1; CD80; costimulatory; adenovirus vector; gene therapy

\section{Introduction}

Recent advances in the understanding of immune responses have revealed that for successful $\mathrm{T}$ cell activation two signals are required. The first is delivered through specific recognition and interaction of $\mathrm{T}$ cell receptor (TCR) with the target $\mathrm{MHC}$-antigenic peptide complex. The second signal is delivered through costimulatory molecules expressed on antigen presenting cells (APCs) binding to their counterpart receptors on $\mathrm{T}$ lymphocytes. Association with these molecules both stabilises the interaction of lymphocyte and APC and transduces costimulatory signals leading to cytokine secretion, proliferation and induction of effector function. Several cell surface molecules such as ICAM-1 and LFA3 have been shown to have a costimulatory function but one of the dominant costimulatory pathways is provided by signalling through CD28. ${ }^{1}$ Two ligands for CD28 have been described, B7-1 and B7-2. These ligands are expressed on the majority of professional APCs such as activated $B$ cells, dendritic cells and macrophages. ${ }^{2-4}$ Both the human and murine B7-1 and B7-2 genes have been cloned and shown to be members of the immunoglobulin gene superfamily. ${ }^{2,5-9}$

The potent costimulatory role of B7-1 has been demonstrated in a number of studies of $\mathrm{T}$ cell activation. Proliferation of $\mathrm{T}$ cells in response to PMA or anti-CD3 can

Correspondence: MG Gilligan

Received 28 November 1997; revised 28 January 1998; accepted 10 February 1998 be increased by the addition of B7-1 transfectants. ${ }^{10,11}$ TCR signalling in the absence of costimulation results in $\mathrm{T}$ cell inactivation or 'anergy' in that clone of T lymphocytes. ${ }^{12}$ This represents an unresponsive state in which subsequent presentation of antigen, even in the presence of adequate costimulation, will fail to elicit a response. Induction of $\mathrm{T}$ cell anergy may be one of the mechanisms by which tumours evade recognition by the immune system. While a variety of tumours are potentially immunogenic displaying both normal levels of MHC molecules and intact antigen presentation capacity, most solid tumours do not express B7-1 or B7-2 and therefore, despite having the capacity to deliver the antigenic signal, render $\mathrm{T}$ cells anergic. The B7/CD28 costimulatory pathway has been exploited to enhance specific immune responses against tumours to generate antitumour immunity. Genetically engineered tumour cells constitutively expressing B7-1 have been shown to be rejected in a variety of murine models, and in some cases protection against further challenge with unmodified tumour has been shown. ${ }^{13-18}$

There is now a considerable body of literature describing studies using B7-1 expressing murine tumour cells, but the data available on the effect of B7-1 gene transfer to human tumour cells (or of other immunotherapeutic genes) is relatively limited. Most of the current attempts to develop cancer vaccines in humans have been directed towards melanoma or renal cell cancer both of which are considered to be relatively immunogenic tumour types. There are comparatively few reports on immunotherapy applied to ovarian and cervical cancers although it is known that these tumours can serve as targets for $\mathrm{T}$ cell responses. ${ }^{19-22}$ 
In this study we have engineered a recombinant, replication defective adenovirus vector carrying a human B71 gene for use in gene therapy for ovarian and cervical cancer. Replication defective adenovirus vectors are in many ways uniquely suited for use in gene therapy. These properties have been extensively reviewed ${ }^{23,24}$ and include the ability to prepare high titre virus capable of efficient infection of a range of proliferating and nonproliferating cell types in vitro and in vivo. We have demonstrated that the recombinant virus described in this study provides an efficient means of achieving B7-1 expression in a number of cervical and ovarian cell lines, as well as primary cultures of ovarian tumour cells. We then demonstrated that B7-1 expression can enhance the immunogenicity of these cells thus meeting a necessary requirement for future development of tumour vaccines by this route.

\section{Results}

\section{B7-1 expression at the cell surface}

In agreement with the literature, the tumour cell lines in this study were found not to express endogenous B7-1 (results not shown). Different concentrations of RAd-B71 were used to infect the ovarian tumour cell line IGROV1 to determine the optimum amount of virus for use in infection experiments. A recombinant adenovirus expressing B7-1 (RAd-B7-1) was constructed (Figure 1; see Materials and methods for further details). A multiplicity of infection (MOI) of 0.3 plaque forming units (p.f.u.) per cell resulted in 31\% of IGROV-1 cells expressing B7-1 although the mean fluorescence intensity (an indicator of protein levels) was low (Figure 2a and c). B7-
1 expression was achieved on $95-100 \%$ of tumour cells at MOI 30 p.f.u. per cell and above (Figure 2a and d). As the MOI was increased, the mean fluorescence rose progressively and did not plateau even at an MOI of 1000 p.f.u. per cell (Figure 2a). A similar pattern of B7-1 expression was seen in the cell lines, OVCAR3 and SKOV3, infected with increasing amounts of virus (data not shown). Figure $3 a$ shows expression of B7-1 as determined by immunofluorescence microscopy of SKOV3 cells fixed $48 \mathrm{~h}$ after infection. Greater than $90 \%$ of SKOV3 cells became visibly B7-1-positive following infection with 30 p.f.u. per cell of RAd-B7-1. Staining appears throughout the cells with a predominant membrane localisation. No detectable expression of B7-1 can be seen on control uninfected cells (Figure $3 b$ ).

\section{Time course of B7-1 expression}

Experiments with ovarian cell line OVCAR3 and early passage ascitic ovarian tumour cells OvCaMG79 (derived from continuous culture of cells from patient 1) infected with RAdB7-1 at an MOI of 30 p.f.u. per cell demonstrated that maximal expression of B7-1 occurred by $48 \mathrm{~h}$ (Figure 4). Levels of expression remained fairly stable for the first 6 days and by day 10, $240 \mathrm{~h}$ after infection, $67.7 \%$ of OVCAR3 cells (Figure $4 \mathrm{a}$ ) and $29.3 \%$ of OvCaMG79 cells (Figure 4b) remained B7-1-positive. The higher percentage of OVCAR3 than OvCaMG79 cells expressing $B 7-1$ at day 10 is probably due to the effect of RAd-B7-1 infection on the growth of these cell lines. Partial inhibition of cell growth was observed with adenoviral infection of OVCAR3 cells while growth of OvCaMG79 appeared to be unaffected. This would result in less dilution of the viral DNA during cell division in
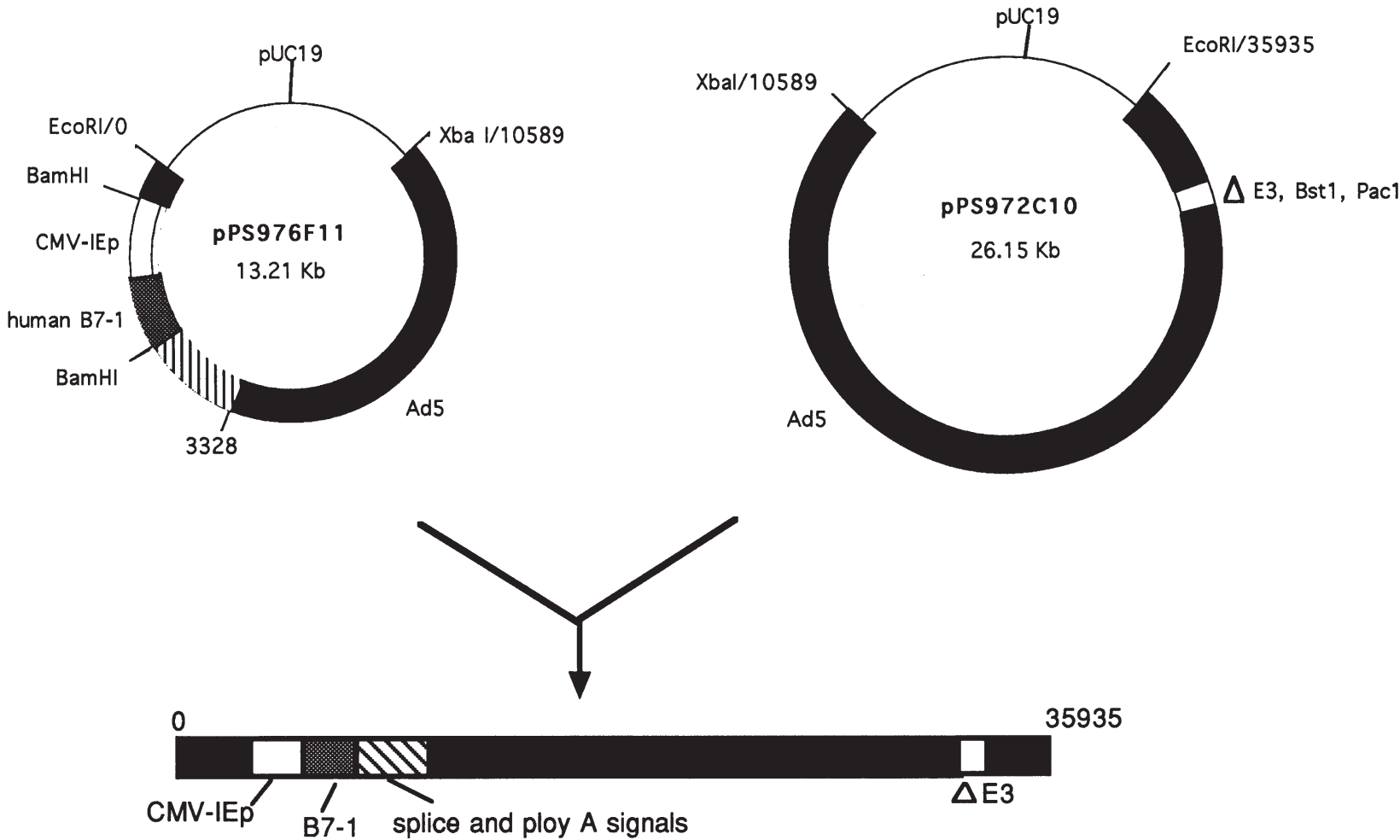

35935

Figure 1 Construction of RAd-B7-1. Plasmids pPS976F11 and pPS972C10 were digested with XbaI, ligated, then a partial EcoRI digest carried out before transfection into 293 cells. Recombinant virus (shown here in a linear form) was rescued from cells by plaque purification. 

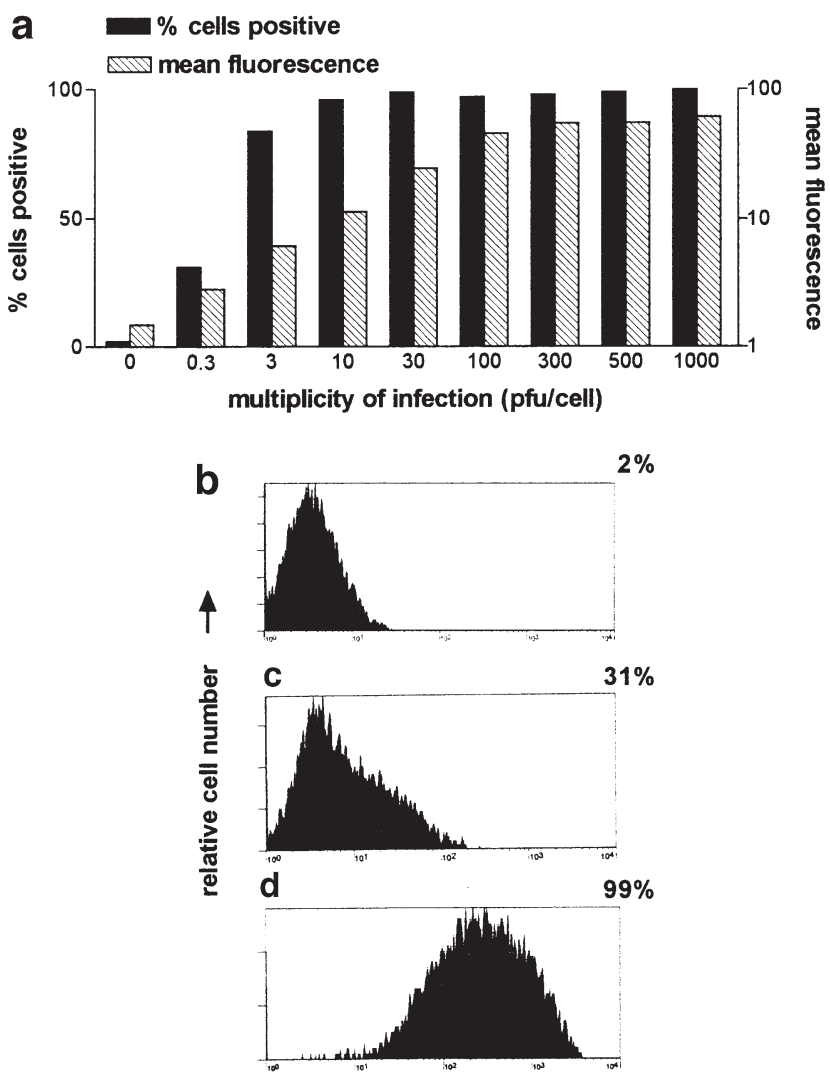

fluorescence intensity $(\log )$

Figure 2 Expression of B7-1 surface glycoprotein on IGROV-1 cells after infection with RAd-B7-1 vector at MOI 0-1000 p.f.u. per cell. Cultures were harvested $48 \mathrm{~h}$ after infection and analysed for cell surface B7-1 expression by FACS. Control uninfected and RAd-B7-1 infected cells were labelled with murine monoclonal antibody BB-1 which recognises human B7-1. Cells were washed then incubated with FITC conjugated goat antimouse antibody. (a) Results are shown as percentage of cells positive $(\square)$ and the log of the mean fluorescence intensity (\$). FACS profiles from control cells (b), cells infected with 0.3 p.f.u. per cell (c) and 30 p.f.u. per cell (d) are shown with the percentage of cells positive noted above. Background fluorescence for control cells was set at $2 \%$ cells positive (b).

OVCAR3 cells and hence a higher percentage of cells remaining positive.

\section{Efficiency of RAd-B7-1 infection of ovarian and cervical tumour cell lines}

RAd-B7-1 virus was used to infect a number of established ovarian and cervical tumour cell lines at an MOI of 30 p.f.u. per cell and cells were assessed for B7-1 expression by FACS analysis $48 \mathrm{~h}$ after infection. A fairly wide range of B7-1 expression at the cell surface was seen between the ovarian and cervical cell lines with most giving between $40-95 \%$ positive cells (Table 1). The ovarian cell line A2780 was shown to be relatively resistant to adenoviral infection as an MOI of 30 p.f.u. per cell resulted in only $4.4 \%$ of cells positive (Table $1 \mathrm{~A}$ ). A high MOI of 500 p.f.u. per cell resulted in $79 \%$ positive cells but with a relatively low mean fluoresence intensity (data not shown). In all cases the number of positive cells and
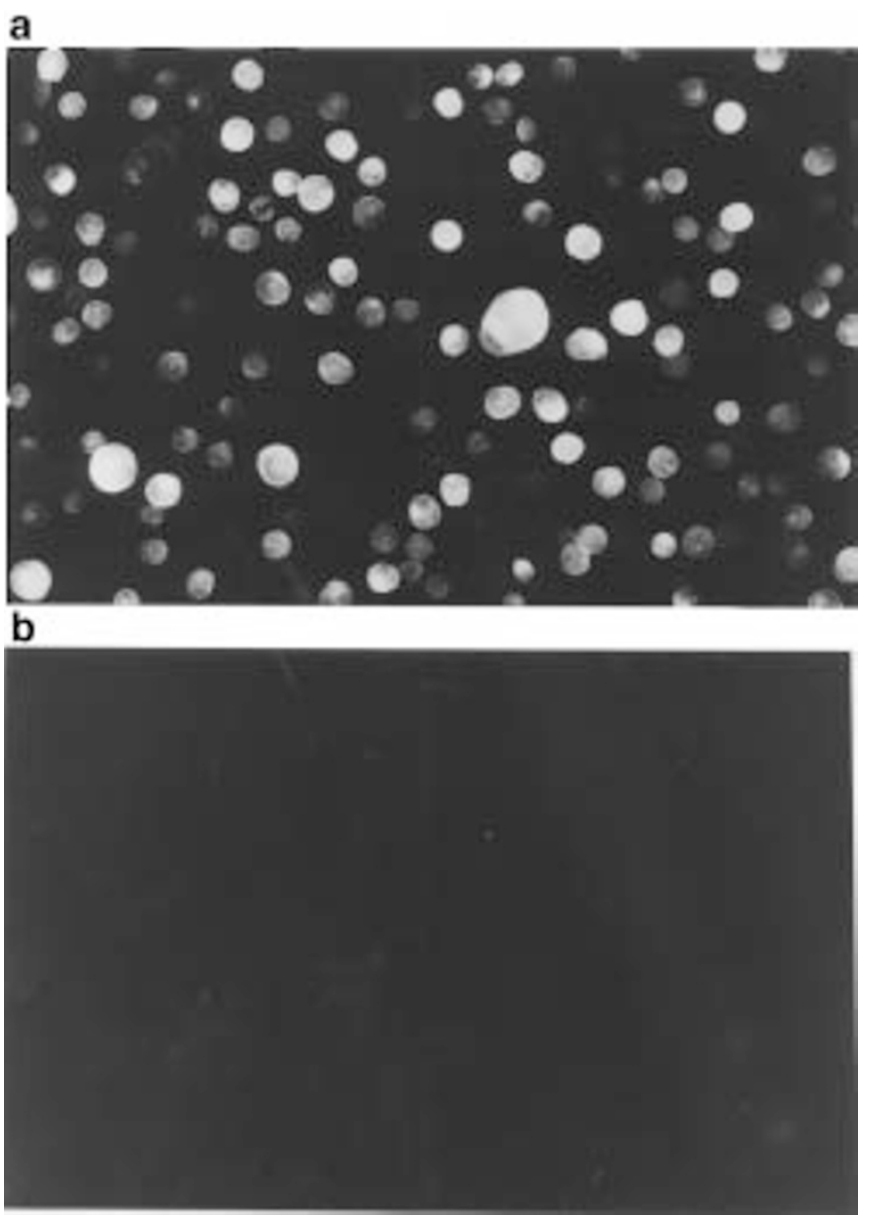

Figure 3 Expression of B7-1 on permeabilised SKOV3 cells $48 \mathrm{~h}$ after infection with RAd-B7-1 vector at an MOI of 30 p.f.u. per cell. Cells were harvested by trypsinisation, washed in PBS then air dried on to multispot slides. Following fixation for $10 \mathrm{~min}$ in acetone:methanol cells were rehydrated then incubated in primary antibody BB-1, which recognises human B7-1. Slides were washed, incubated with FITC conjugated goat antimouse then mounted in DABCO. (a) Cells infected at an MOI of 30 incubated with BB-1 antibody; (b) control uninfected cells incubated with BB-1 antibody.

the amount of B7-1 protein produced could be increased by increasing the amount of virus used during infection (data not shown).

\section{RAd-B7-1 infection of primary ovarian tumour cells}

Having demonstrated that the established cell lines are susceptible to adenovirus infection we next examined the infectivity of primary ovarian tumour cells. Tumour cells directly cultured from the ascitic fluid of seven patients with adenocarcinoma of the ovary were studied for the expression of a number of different cellular markers in order to verify cell type. Markers studied included MHC class I and II, p53, CA125, cytokeratin, epithelial membrane antigen, vimentin, HER2/neu, CEA and 5T4. In all cases, the use of these markers confirmed cell cultures consisting of greater than $90 \%$ tumour cells (data not shown). Tumour cells infected with RAd-B7-1 at an MOI of 30 p.f.u. per cell gave high levels of B7-1 expression 

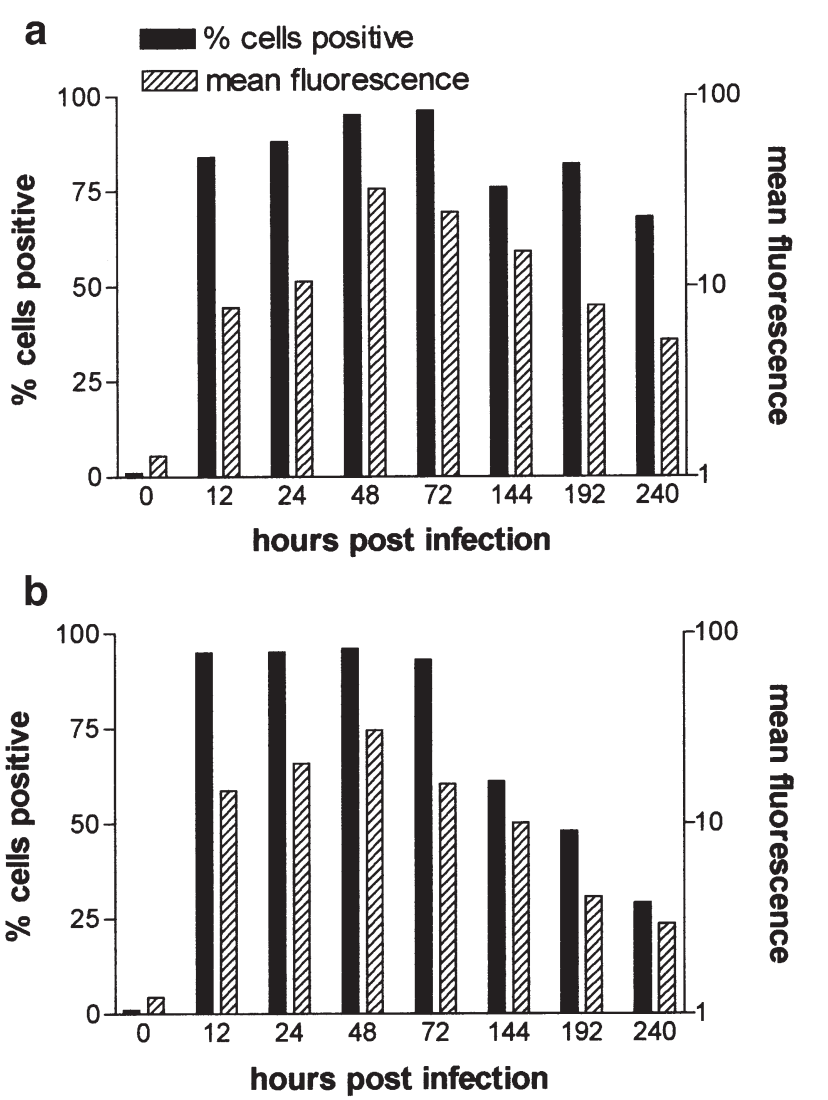

Table 1 Expression of B7-1 on ovarian and cervical carcinoma cell lines infected with RAd-B7-1
Cell lines
$\%$ Positive cells

A. Ovarian carcinoma cell lines

$\begin{array}{lr}\text { Caov-3 } & 39 \\ \text { OVCAR3 } & 76 \\ \text { IGROV-1 } & 79 \\ \text { OAW28 } & 59 \\ \text { OAW42 } & 66 \\ \text { 59M } & 27 \\ \text { 41M } & 47 \\ \text { A2780 } & 4 \\ \text { SKOV3 } & 91\end{array}$

B. Cervical carcinoma cell lines

$\begin{array}{ll}\text { C-33A } & 77 \\ \text { C-4I } & 44 \\ \text { HT-3 } & 80 \\ \text { ME-180 } & 59 \\ \text { MS751 } & 96 \\ \text { SiHa } & 88 \\ \text { HeLa } & 84 \\ \text { CaSki } & 79\end{array}$

a Surface expression of B7-1 on ovarian (A) and cervical (B) carcinoma cell lines infected with RAd-B7-1 virus at an MOI of 30 p.f.u. per cell. Cells were harvested $48 \mathrm{~h}$ after infection and analysed for B7-1 expression by FACS. Control uninfected and RAd-B7-1 infected cells were labelled with the human B7-1-specific BB-1 monoclonal antibody as described in Materials and methods. Uninfected cells were negative for B7-1 staining and RAd-B7-1-infected cells stained negative using an isotypematched control antibody. Results are shown as the percentage of cells positive and are typical of those obtained in three separate experiments. ing infection at an MOI of 30 p.f.u. per cell of RAd-B7-1 vector. Cultures were harvested at various time-points following infection and analysed for B7-1 surface expression by FACS. Control uninfected and RAd-B7-1 infected cells were labelled with murine monoclonal antibody BB-1 which recognises human B7-1. Cells were washed then incubated with FITC conjugated goat antimouse antibody. (a) OVCAR3 cells (b) OvCaMG79 early passage ascitic ovarian tumour cells. Results are shown as percentage of

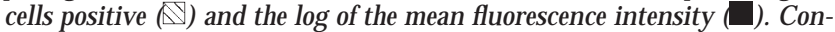
trol values for background fluorescence are shown at time zero. The results of a representative experiment are shown.

at the cell surface in six out of seven cases (Table 2). In the case of patient 4 where only $11 \%$ of cells were found to be positive, B7-1 expression could be increased to $60 \%$ of cells when an MOI of 300 p.f.u. per cell was used.

\section{Increased immunogenicity of tumour cells expressing B7-1}

To address the question of whether tumour cells modified to express B7-1 are more immunogenic, PBLs from three healthy donors were stimulated with an ovarian tumour cell line and a cervical cell line infected with RAd-B7-1 or control adenovirus RAd35. Cells were incubated with IFN $\gamma$ following infection and this was shown by flow cytometry to upregulate both MHC class I and class II expression (data not shown). Figure 5a-c shows the proliferative response obtained from three donors using the established ovarian carcinoma cell line OVCAR3 as stimulators in an MLTC. Control cells induce

Table 2 Expression of B7-1 on primary ovarian tumour cells infected with RAd-B7-1

Primary ovarian
tumour cells

Patient 1

Patient 2

Patient 3

Patient 4

Patient 5

Patient 6

Patient 7

95
54
90
11
95
74
84

aSurface expression of B7-1 on primary ovarian tumour cells isolated from the ascitic fluid of patients with ovarian cancer and infected with RAd-B7-1 vector at an MOI of 30 p.f.u. per cell. Cells were harvested $48 \mathrm{~h}$ after infection and the percentage of cells expressing B7-1 determined by FACS.

a low level of $\mathrm{T}$ cell proliferation while cells expressing B7-1 induced a strong proliferative response that was dependent on the number of tumour cells. A slightly different pattern of proliferation was seen when cervical tumour cells HeLa were used as stimulators (Figure $5 d-f)$. In this case an increase in proliferation was seen with up to $1 \times 10^{4}$ stimulator cells, but thymidime 

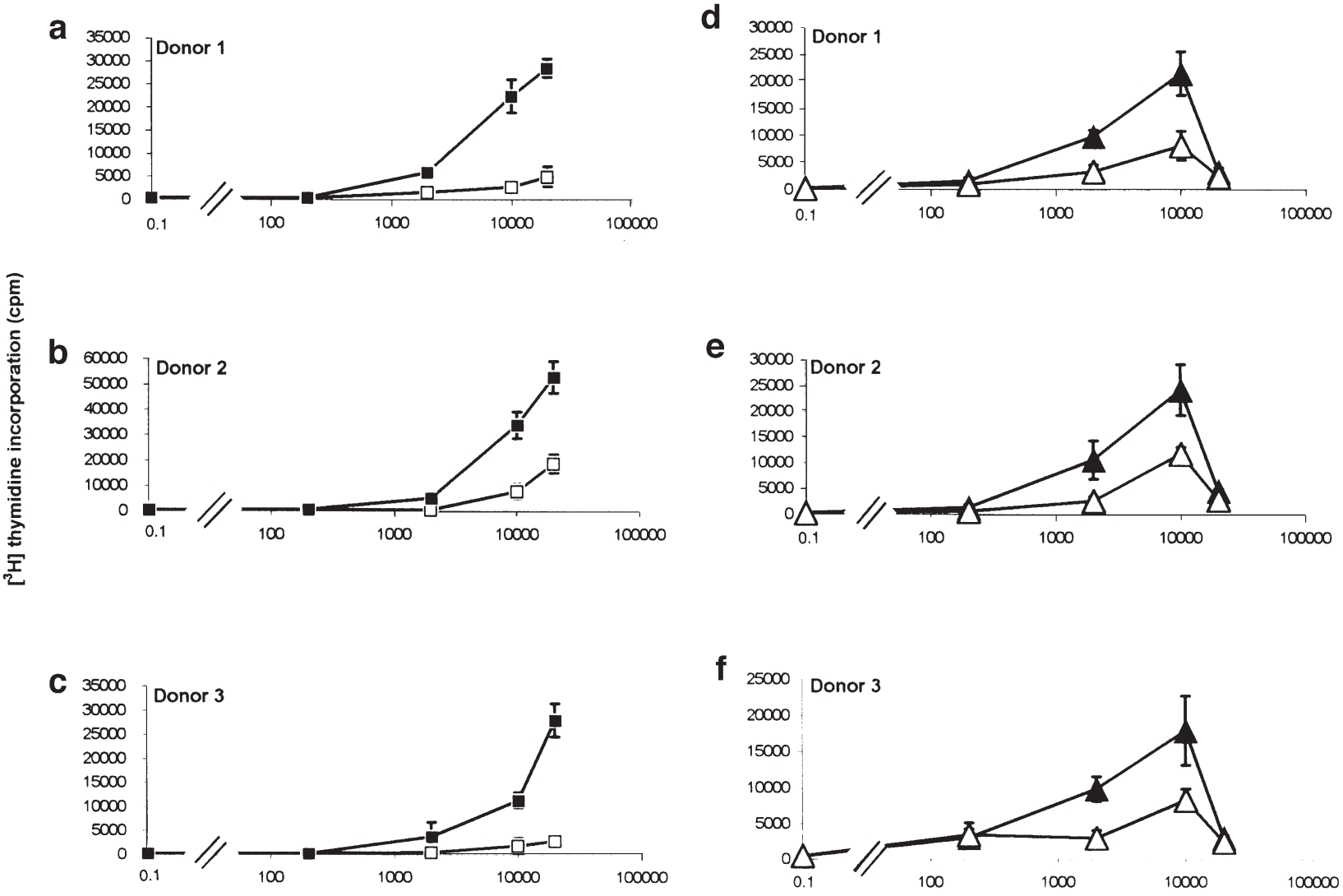

Number of Stimulators

Figure 5 Increased proliferation of allogeneic PBLs stimulated with B7-1 positive tumour cells. $(a-c)$ Proliferation of PBLs from three donors after 6-day culture with irradiated OVCAR3 cells. $\square$ Cells preinfected with RAd35; cells preinfected with RAd-B7-1. (d-f) Proliferation of PBLs from three donors after 6-day culture with irradiated HeLa cells. $\triangle$ Cells preinfected with RAd35, $\boldsymbol{\Delta}$ cells preinfected with RAd-B7-1. Each point represents the mean ( \pm s.e.m.) of six determinations. The results shown are typical of those found in three separate experiments.

uptake was lower with $2 \times 10^{4}$ stimulator cells. This decrease in proliferation was seen in both control cultures and cultures stimulated with RAd-B7-1-infected HeLa cells.

The MLTC experiment was repeated using freshly isolated ascitic ovarian tumour cells OTCMG61 (Figure 6ac) and an early passage cell culture derived from a solid ovarian tumour OvCaMG75 (Figure 6d-f). Ascitic ovarian tumour cells were infected at an MOI of 30 p.f.u. per cell of RAd-B7-1 or RAd35 while those from the solid tumour were infected at an MOI of 300 p.f.u. per cell (57.6\% of cells positive). As seen in Figure 6, the same pattern of response is seen using PBLs from three donors, that is an increased proliferative response to cells expressing B7-1 compared with control. That the increased $\mathrm{T}$ cell proliferation was due to the expression of B7-1 was confirmed by use of neutralising anti-B7-1 antibody added at the initiation of the MLTC culture (Figure 7). Complete inhibition of excess proliferation relative to control cells was achieved by the addition of $2.5 \mu \mathrm{g} / \mathrm{ml}$ of BB-1 antibody. Addition of an equal concentration of isotype control antibody did not reduce the B7-
1 enhanced proliferation, and none of the antibodies had any effect on control values.

\section{Discussion}

The present investigation was undertaken to determine if an adenoviral vector would be an efficient agent to use for gene transfer of B7-1 in the modification of ovarian and cervical tumour cells for use in immunotherapy. Previously B7-1 has been expressed in several tumour cell lines following transfection or retroviral transfer of DNA followed by drug selection of positive cells. The low titre obtained from retroviral vectors, their ability to infect only dividing cells and the consequent requirement for multiple cycles of infection, or for drug selection of transduced cells, limits their application for modification of tumour cells for gene therapy, particularly for direct in vivo viral delivery approaches. These vectors may be suitable for modification of tumour cells without extended culture ex vivo as Jaffee et $a l^{25}$ reported high efficiency retroviral gene transfer into a number of primary human tumour explants without drug selection. Sule-Suso et al ${ }^{26}$ 
a

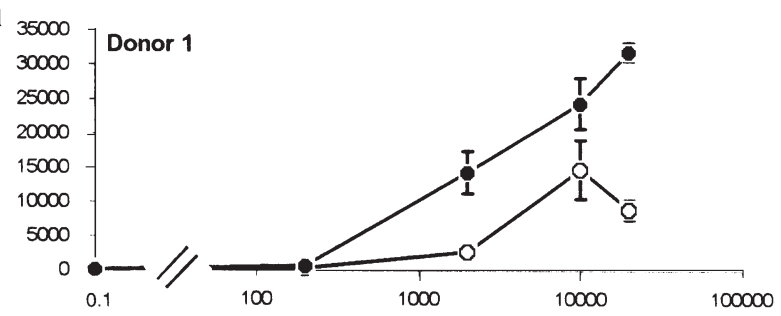

d

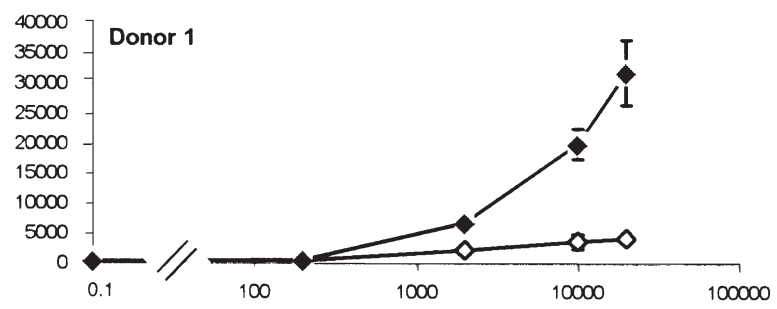

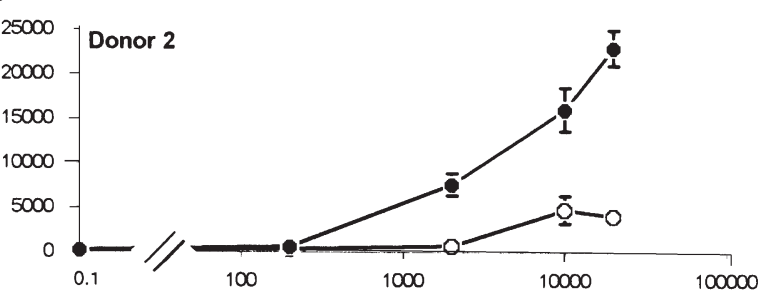

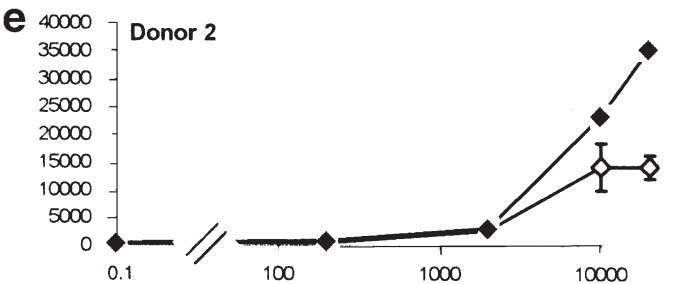

100000

C

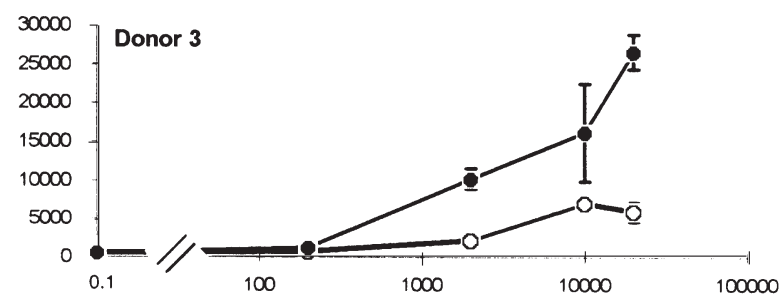

f

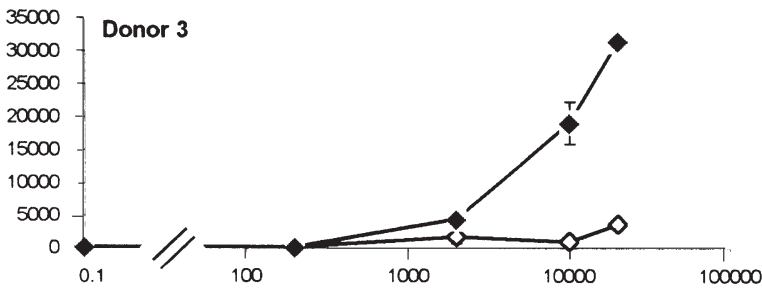

Number of Stimulators

Figure 6 Increased proliferation of allogeneic PBLs stimulated with B7-1 positive primary ovarian tumour cells. (a-c) Proliferation of PBLs from three donors after 6-day culture with irradiated ascitic ovarian tumour cells (patient 2, OTCMG61). $\bigcirc$ Cells preinfected with RAd35; 0 cells preinfected with RAd-B7-1, MOI 30 p.f.u. per cell. (d-f) Proliferation of PBLs from three donors after 6-day culture with irradiated early passage ovarian tumour cells OvCaMG75 derived from a solid tumour. $\diamond$ Cells preinfected with RAd35; $\bullet$ cells preinfected with RAd-B7-1, MOI 300 p.f.u. per cell. Each point represents the mean $( \pm$ s.e.m.) of six determinations. The results shown are typical of those found in three separate experiments.

transfected and selected a cloned melanoma cell line with B7-1, and showed increased stimulation of both autologous and allogeneic PBLs compared with parental unmodified melanoma cells. An increase in the cytotoxic activity of allogeneic PBLs was also described. Dohring et $a l^{27}$ used a retroviral vector to deliver B7-1 to a melanoma, ovarian and myelomonocytic leukemia cell line. Cells were selected for G418 resistance then used to stimulate allogeneic PBLs. Increases in proliferation were seen with all three lines, and the expanded $\mathrm{T}$ cells showed efficient lysis of the wild-type unmodified tumour cells. Previous studies have also depended on the establishment of long-term cultures of tumour cells which, in the context of developing a clinically useful cancer vaccine, is time-consuming and often inefficient, possibly resulting in the loss of critical tumour antigens..$^{28}$ This study describes the construction and application of a recombinant B7-1 adenovirus. This vector can rapidly and efficiently infect ovarian and cervical tumour cell lines with B7-1 expression detectable at the cell surface within $12 \mathrm{~h}$ following infection. Expression is stable and detectable up to 10 days as determined by a time course of gene expression. Constitutive gene expression of B7-1 is not required for the effector phase of $\mathrm{T}$ cell lysis since B7-CD28 interaction is crucial in the induction phase of a cytotoxic response but not during the lytic phase when unmodified cells may be recognised and killed. ${ }^{29} \mathrm{~A}$ range of virus dilutions was used to determine the optimal MOI for use in experiments and to study the effect of adding increasing amounts of virus. High efficiencies of gene transfer and expression ( $>95 \%$ IGROV-1 tumour cells positive) could be obtained using an MOI of 30 p.f.u. per cell. Although an MOI of 30 p.f.u. per cell resulted in a maximal number of cells positive the amount of protein expressed at the cell surface could be increased by increasing the MOI. When a number of ovarian and cervical cell lines were infected at an MOI of 30 most showed efficient gene delivery (27-96\% cells positive). As well as efficiently infecting cell lines this vector was also able to transduce primary cultures of ovarian tumour cells with similar high efficiency in six out of seven cases.

An approach that did not rely upon culture of tumour 


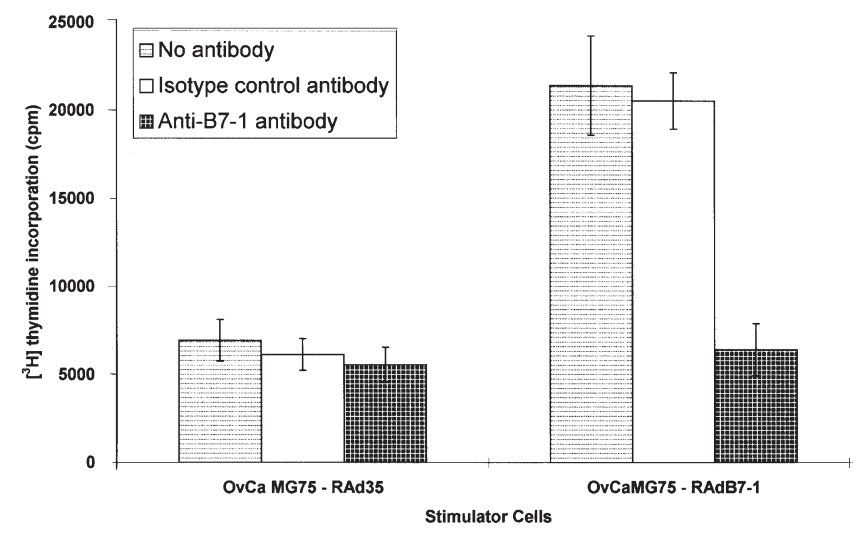

Figure 7 Antibody blocking of B7-1-mediated enhanced T cell proliferation. Early passage ovarian tumour cells OvCaMG75 infected with RAd35 or RAd-B7-1 adenovirus (MOI 300 p.f.u. per cell) were irradiated, then $10^{4}$ tumour cells incubated with $10^{5}$ allogeneic PBLs for 6 days. No antibody, an isotype control antibody or antibody recognising human B7$1(2.5 \mu \mathrm{g} / \mathrm{ml})$ were included in cultures. Results of a representative experiment are shown.

cells would be the most convenient method for delivering therapeutic genes. We have found that suspensions of ovarian tumour cells within $24 \mathrm{~h}$ of separation from ascitic fluid or immediately following removal from liquid nitrogen could also be infected with RAd-B7-1. These tumour cells are present as clumps of cells and therefore an accurate MOI is difficult to achieve and cells cannot be FACS analysed, but immunofluorescence staining has shown gene transfer and expression (data not shown). These results are similar to those reported by Dessureault et al ${ }^{30}$ who showed efficient B7-1 gene transfer to a number of cell lines and dissociated primary tumours using a recombinant adenovirus. This study did not include ovarian tumour cells and the immunogenicity of modified cells was not reported. The mechanism underlying the resistance of some tumour cells (particularly ovarian cell line A2780) to infection with adenovirus is under investigation. A2780 cells express high levels of MHC class I and the integrin vitronectin molecules (data not shown) important for virus attachment and internalisation, suggesting that the mechanism underlying resistance lies elsewhere.

The capacity of tumour cells expressing B7-1 to stimulate allogeneic PBLs was determined by a mixed lymphocyte tumour cell assay. Tumour cells expressing B7-1 induced significantly higher levels of proliferation than control cells and this was found to be directly attributable to B7-1 as antibodies against this molecule were found to block the response. Similar levels of proliferation (twoto six-fold compared with control cultures) to those reported here for the cervical tumour cell line HeLa have been reported by Kaufmann et al ${ }^{31}$ using stably transfected B7-1 clones of cervical tumour cells as stimulators. The decrease in proliferation reported here when $2 \times 10^{4}$ stimulator HeLa cells were used was not detected by this group as their studies were carried out with $1 \times 10^{4}$ stimulators. A possible explanation for this effect could be that HeLa cells secrete low amounts of soluble T cell inhibitory factors such as TGF- $\beta$ or interleukin- $10^{32}$ and
Adenoviral delivery of B7-1

the suppressive effects only become apparent in the MLTC when sufficient tumour cells are present. To determine if an increased proliferative response was seen using freshly isolated tumour cells rather than established cell lines, ascitic ovarian tumour cells and early passage cultures from a solid ovarian tumour were modified to express B7-1 then used as stimulators in mixed lymphocyte tumour cell cultures. Primary cells gave a pattern of response similar to that seen using established cell lines. In addition to the proliferation data shown in this report, a number of the other cervical and ovarian cell lines, as well as primary ovarian tumour cells have been used in the MLTC assay and increased proliferation to B7-1 expressing cells has always been detected (data not shown). Although this assay confirms that the B7-1 molecule is biologically active and that ovarian and cervical tumour cells are immunogenic the issue of antigenicity is not addressed. This would need to be studied in an autologous setting through generation of a specific $\mathrm{CD}^{+}$and/or $\mathrm{CD} 8^{+} \mathrm{T}$ cell response. It is already well recorded that a variety of tumour cells express specific antigens including melanoma, ${ }^{33-35}$ ovary, ${ }^{20-22}$ breast, ${ }^{22,36}$ and cervical carcinoma. ${ }^{37}$ It therefore appears possible that B7-1 transfer into tumour cells could be used as a means to generate a cellular vaccine which could then be used to elicit tumour-specific CTL response. When considering the extension of the present investigation to its clinical application, the possible combined use of cytokine genes, in particular IL-2 will need to be explored. This is in the light of a number of animal studies which have reported more effficient tumour regression and systemic immunity using both B7-1 and IL-2 in combination rather than either gene on its own. ${ }^{38,39}$

In conclusion, the results reported here demonstrate that the adenoviral vector is a rapid and efficient means of gene delivery to ovarian and cervical tumour cells. The results demonstrate that B7-1 expression in human ovarian and cervical tumour cells increases their immunogenicity, as evaluated using proliferation of allogeneic PBL. These data support the rationale for potential use of adenovirally delivered B7-1 for genetic immunotherapy of ovarian and cervical cancer.

\section{Materials and methods}

\section{Preparation of primary ovarian tumour cells}

Malignant ascites were obtained from patients with epithelial adenocarcinoma of the ovary. Heparinised ascitic fluid was centrifuged at $800 \mathrm{~g}$ for $15 \mathrm{~min}$ and the cell pellet resuspended in serum-free RPMI 1640 culture medium. Live cells were separated from red blood cells and dead cells by centrifugation through Ficoll-Hypaque (Lymphoprep; Nycomed Pharma, Oslo, Norway). Tumour cells were then separated from mononuclear cells by centrifugation on $75 / 100 \%$ discontinuous FicollHypaque gradients. ${ }^{20}$ Ovarian tumour cells were maintained in culture in DME medium supplemented with $10 \%$ foetal bovine serum (FBS), $1 \%$ glutamine, $1 \%$ penicillin and streptomycin, $1 \%$ nonessential amino acids (Life Technologies, Paisley, UK)) and $10 \mu \mathrm{g} / \mathrm{ml}$ insulin (Sigma, Poole, UK).

Ovarian cell line OvCaMG75 was generated from an epithelial ovarian tumour by collagenase digestion. In 
brief, the tumour was minced manually into small fragments then enzymatically dispersed to yield a single cell suspension by digestion for $4 \mathrm{~h}$ in 3\% collagenase type I (Sigma), 0.02\% DNase (Sigma) in serum-free DME plus $20 \mathrm{~mm}$ hepes and $1 \%$ penicillin and streptomycin. Tumour cells were cultured in 6-cm Petri dishes preseeded with a confluent monolayer of irradiated NIH3T3 cells (60 Gy, cobalt 60) to prevent the outgrowth of fibroblasts. OvCaMG75 cells were grown in RPMI 1640 supplemented with $1 \%$ glutamine, $1 \%$ penicillin and streptomycin, and 10\% FBS.

\section{Tumour cell lines}

Human cervical tumour cell lines SiHa, MS751, ME-180, HT-3, C-33A, C-4I and ovarian tumour cell lines OVCAR3 and Caov3 were obtained from the American Type Culture Collection (Rockville, MD, USA). OAW28, OAW42, 59M and 41M ovarian tumour cell lines were provided by Dr G Ganeshan, John Radcliffe Hospital, Oxford, UK. IGROV-1 cells were kindly provided by Dr Martin Ford, Glaxo Wellcome Research Laboratories, Stevenage, UK. The ovarian cell line A2780 was provided by Dr J Plumb, Glasgow University, Glasgow, UK. The above cervical cell lines were grown in RPMI 1640 supplemented with 1\% glutamine and 10\% FBS. Cervical tumour cells CaSki and HeLa were cultured in DME supplemented with 1\% glutamine and 10\% FBS. Apart from IGROV-1 and A2780 cells which were grown in RPMI1640 , the ovarian cell lines were grown in DME supplemented as above. Cell lines $59 \mathrm{M}$ and $41 \mathrm{M}$ required in addition supplements of $10 \mu \mathrm{g} / \mathrm{ml}$ insulin and $1 \mathrm{~mm}$ sodium pyruvate (Life Technologies). Cell line OVCAR3 required the addition of $1 \%$ nonessential amino acids and $10 \mu \mathrm{g} / \mathrm{ml}$ insulin. All cell lines were subcultured weekly and routinely checked for mycoplasma contamination.

\section{Construction of recombinant Ad-CMV-B7-1 virus (RAd- B7-1)}

Human B7-1 cDNA was generously provided by Dr M Jenkins (Minnesota, MN, USA) in the expression vector pcEXV-hB7. ${ }^{11}$ This was cut with EcoRI and the B7 fragment (919 bp) gel-purified and inserted downstream of the CMV immediate-early promoter in the retroviral vector plasmid pLNCX (kindly provided by AD Miller, Fred Hutchinson Cancer Research Center, Seattle, WA, USA) to yield plasmid pxLNC-hB7. Plasmid pPS971C5 contains the first part of the adenovirus sequence up to the $\mathrm{XbaI}$ site at 10589 base pairs, but has been deleted for the whole of the E1a and the majority of the EIb region of Ad5. The deleted region is replaced by a unique Bam $\mathrm{HI}$ cloning site, and by splice and poly A/termination signals from the human beta globin gene and the human complement C2 genes, respectively. The human B7-1 was subcloned together with the CMV immediate-early promoter into the BamHI site to give pPS976F11. Plasmid pPS972C10 encompasses the rest of the adenoviral genome from bp 10589, to the right end, except for a $1878 \mathrm{bp}$ deletion between two XbaI sites in the E3 region (28592 to 30470), which now contains a short polylinker with unique BstBI and PacI sites. Plasmids pPS976F11 and pPS972C10 were linearised with $\mathrm{XbaI}$, ligated together and then partially digested with EcoRI to break up large concatamers, before being transfected by calcium phosphate precipitation into 293 cells. ${ }^{40}$ Recombinant virus was rescued, plaque purified (Figure 1), grown and titrated as described ${ }^{41}$ to give virus stocks for experiments. Recombinant adenovirus RAd35 (an E1/E3 deleted adenovirus carrying the E. coli LacZ gene, ${ }^{42}$ kind gift of Dr Gavin Wilkinson, Cardiff University, UK) was used as a control vector.

\section{Infection of tumour cells}

RAd-B7-1 was used to infect ovarian and cervical cell lines, and primary cultures of ovarian tumour cells derived from ascites and solid tumour. Tumour cells were cultured to between $50-70 \%$ confluence in $6-\mathrm{cm}$ plates; then infection with the recombinant adenovirus was performed in $0.5 \mathrm{ml}$ medium plus $10 \% \mathrm{FBS}$ at a MOI of between 3 and 1000 p.f.u. per cell for $2 \mathrm{~h}$ with occasional agitation. The virus containing medium was removed, replaced by growth medium and cells were harvested by trypsinisation at intervals to study B7-1 expression.

\section{Cell staining and flow cytometry}

For visualisation of B7-1 expression, trypsinised cells were washed in PBS Dulbecco's A, air-dried for $2 \mathrm{~h}$ on to microspot slides then fixed in a 1:1 mix of acetone:methanol for $10 \mathrm{~min}$. Following rehydration in DPBS, cells were stained for $60 \mathrm{~min}$ at room temperature with primary antibody BB-1, a murine antibody recognising human B7-1 (Becton Dickinson, Oxford, UK) at a concentration of $10 \mu \mathrm{g} / \mathrm{ml}$. Cells were counterstained with FITC-conjugated goat antimouse antibody (Sigma) for 30 min at room temperature, mounted in DABCO then viewed using a Nikon UV-fluorescense microscope (Nikon, Tokyo, Japan). For flow cytometry cells were harvested by trypsinisation then stained for $60 \mathrm{~min}$ at $4^{\circ} \mathrm{C}$ with primary antibody BB-1, $10 \mu \mathrm{g} / \mathrm{ml}$. Cells were counterstained with FITC conjugated goat antimouse antibody for $30 \mathrm{~min}$ at $4^{\circ} \mathrm{C}$. Background fluorescence for control staining in FACS assays was set by staining uninfected cells with BB-1 antibody and typically yielded $2 \%$ cells positive. No significant differences in control values were detected when cells were stained with an isotype matched irrelevant antibody or when cells were infected with control RAd35 vector before staining (data not shown). Following fixation in $1 \%$ paraformaldehyde, cells were analysed on a Coulter XL flow cytometer (Coulter, FL, USA) with standard filter configuration.

\section{Mixed lymphocyte tumour cell cultures (MLTC)}

Tumour cells to be used as stimulators were infected with RAd35 or RAd-B7-1 then incubated with $150 \mathrm{U} / \mathrm{ml}$ interferon gamma (IFN $\gamma$; R\&D Systems, Oxford, UK), for $48 \mathrm{~h}$. Following IFN $\gamma$ treatment cells expressed high levels of HLA class I and class II as determined by immunocytochemistry and flow cytometry (data not shown). Stimulator cells were harvested by trypsinisation then irradiated at 6000 rad (cobalt 60). Peripheral blood lymphocytes (PBLs) were obtained from normal laboratory donors by separation down Ficoll-Hypaque. $10^{5}$ PBLs were cultured in 96 well round bottomed plates for 
6 days with between $2 \times 10^{2}$ to $2 \times 10^{4}$ irradiated tumour cells. Assays were set up using RPMI 1640 medium supplemented with $1 \%$ glutamine and $10 \%$ human serum (AB serum; Sigma) in the absence of IL-2. Proliferation was determined by adding $0.5 \mu \mathrm{Ci}{ }^{3} \mathrm{H}$-thymidine (Amersham International, Buckinghamshire, UK) to each well for the last $18 \mathrm{~h}$ of culture. Cells were harvested on a Skatron plate harvester and ${ }^{3} \mathrm{H}$-thymidine uptake measured by liquid scintillation counting. The mean of six wells was determined for each stimulation, and for control PBLs alone. Inhibition of signalling through B7-1 was performed by addition of $2.5 \mu \mathrm{g} / \mathrm{ml}$ of antibody to B7 (BB-1) or an isotype control at the initiation of co-culture. The optimum amount of antibody was determined by titration experiments (data not shown).

\section{Acknowledgements}

The authors would like to thank Kim Ward for performing all the FACS analysis presented in this study. The study was supported by the Medical Research Council UK.

\section{References}

1 Lenshow DJ, Walunas TL, Bluestone JA. CD28/B7 system of T cell costimulation. Annu Rev Immunol 1996; 14: 233-258.

2 Freeman GJ et al. Structure, expression and T cell costimulatory activity of the murine homologue of the B lymphocyte activation antigen B7. J Exp Med 1991; 174: 625-631.

3 Larsen $\mathrm{CP}$ et al. Functional expression of the costimulatory molecule, B7/BB1, on murine dendritic cell populations. J Exp Med 1992; 176: 1215-1220.

4 Razi-Wolf $\mathrm{Z}$ et al. Expression and function of the murine B7 antigen, the major costimulatory molecule expressed by peritoneal exudate cells. Proc Natl Acad Sci USA 1992; 89: 4210-4214.

5 Azuma $\mathrm{M}$ et al. B70 antigen is a second ligand for CTLA-4 and CD28. Nature 1993; 366: 76-79.

6 Chen C, Gault A, Shen L, Nabavi N. Molecular cloning and expression of early $\mathrm{T}$ cell costimulatory molecule- 1 and its characterisation as B7-2 molecule. J Immunol 1994; 152: 49294936.

7 Freeman GJ et al. B7 a new member of the Ig superfamily with unique expression on activated and neoplastic B cells. J Immunol 1989; 143: 2714-2722.

8 Freeman GJ et al. Cloning of B7-2: a CTLA-4 counter receptor that costimulates human T cell proliferation. Science 1993; 262: 909-911.

9 Freeman GJ et al. Murine B7-2, an alternative CTLA4 counterreceptor that costimulates $\mathrm{T}$ cell proliferation and interleukin2 production. J Exp Med 1993; 178: 2185-2195.

10 Gimmi CD et al. B cell surface antigen B7 provides a costimulatory signal that induces $\mathrm{T}$ cells to proliferate and secrete interleukin2. Proc Natl Acad Sci USA 1991; 88: 6575-6579.

11 Norton SD et al. The CD28 ligand B7, enhances IL-2 production by providing a costimulatory signal to T cells. J Immunol 1992; 149: $1556-1561$.

12 Schwartz RH. A cell culture model for T lymphocyte clonal anergy. Science 1990; 248: 1349-1356.

13 Townsend SE, Allison JP. Tumour rejection after direct costimulation of $\mathrm{CD}^{+} \mathrm{T}$ cells by B7-transfected melanoma cells. Science 1993; 259: 368-370.

14 Baskar S et al. Constitutive expression of B7 restores immunogenicity of tumour cells expressing truncated major histocompatibility complex class II molecules. Proc Natl Acad Sci USA 1993; 90: 5687-5690.
15 Baskar S et al. Major histocompatibility complex class II+B7-1+ tumour cells are potent vaccines for stimulating tumour rejection in tumour-bearing mice. J Exp Med 1995; 181: 619-629.

16 Townsend S, Su F, Atherton J, Allison J. Specificity and longevity of antitumour immune responses induced by B7-transfected tumours. Cancer Res 1994; 54: 6477-6483.

17 Chen $\mathrm{L}$ et al. Tumour immunogenicity determines the effect of B7 costimulation on $\mathrm{T}$ cell-mediated tumour immunity. J Exp Med 1994; 179: 523-532.

18 Chen L et al. B7-1/CD80 transduced tumour cells elicit better systemic immunity than wild-type tumour cells admixed with Corynebacterium parvum. Cancer Res 1994; 54: 5420-5423.

19 Borysiewicz LK et al. A recombinant vaccinia virus encoding human papillomavirus types 16 and 18, E6 and E7 proteins as immunotherapy for cervical cancer. Lancet 1996; 347: 1523-1527.

20 Ioannides CG et al. Tumour cytolysis by lymphocytes infiltrating ovarian malignant ascites. Cancer Res 1991; 51: 4257-4265.

21 Ioannides CG et al. Cytotoxic T cell clones isolated from ovarian tumour-infiltrating lymphocytes recognise multiple antigenic epitopes on autologous tumour cells. J Immunol 1991; 146: 1700-1707.

22 Peoples GE et al. Breast and ovarian cancer-specific cytotoxic T lymphocytes recognise the same HER2/neu-derived peptide. Proc Natl Acad Sci USA 1995; 92: 432-436.

23 Berkner KL. Development of adenovirus vectors for the expression of heterologous genes. Biotechniques 1988; 6: 616-629.

24 Kozarsky KF, Wilson JM. Gene therapy: adenovirus vectors. Curr Opin Genet Dev 1993; 3: 499-503.

25 Jaffee EM et al. High efficiency gene transfer into primary human tumour explants without cell selection. Cancer Res 1993; 53: $2221-2226$.

26 Sule-Suso J et al. A B7-1 transfected human melanoma line stimulates proliferation and cytotoxicity of autologous and allogeneic lymphocytes. Eur J Immunol 1995; 25: 2737-2742.

27 Dohring C, Angman L, Spagnoli G, Lanzavecchia A. T helper and accessory cell independent cytotoxic responses to human tumour cells transfected with a B7 retroviral vector. Int J Cancer 1994; 57: 754-759.

28 Knuth A et al. Cytotoxic $\mathrm{T}$ cell clones against an autologous human melanoma: specificity study and definition of three antigens by immunoselection. Proc Natl Acad Sci USA 1989; 86: 2804-2808.

29 Harding FA, Allison JP. CD28-B7 interactions allow the induction of $\mathrm{CD} 8^{+}$cytotoxic T-lymphocytes in the absence of exogenous help. J Exp Med 1993; 177: 1791-1796.

30 Dessureault S, Graham F, Gallinger S. B7-1 transfer into human cancer cells by infection with an adenovirus B7-1 (Ad-B7) expression vector. Ann Surg Oncol 1996; 3: 317-324.

31 Kaufmann AM et al. Expression of CD80 enhances immunogenicity of cervical carcinoma cells in vitro. Cell Immunol 1996; 169: $246-251$.

32 Sulitzzeanu D. Immunosuppressive factors in human cancers. Adv Cancer Res 1993; 60: 247-267.

33 Boon $\mathrm{T}$ et al. Tumour antigens recognised by $\mathrm{T}$ lymphocytes. Annu Rev Immunol 1994; 12: 337-365.

34 Kawakami $\mathrm{Y}$ et al. Cloning of the gene coding for a shared human melanoma antigen recognised by autologous $\mathrm{T}$ cells infiltrating into tumour. Proc Natl Acad Sci USA 1994; 91: 3515-3519.

35 Kawakami $Y$ et al. Identification of a human melanoma antigen recognised by tumour-infiltrating lymphocytes associated with in vivo tumour rejection. Proc Natl Acad Sci USA 1994; 91: 6458-6462.

36 Linehan DC et al. Tumour-specific and HLA-A2-restricted cytolysis by tumour-associated lymphocytes in human metastatic breast cancer. J Immunol 1995; 155: 4486-4491.

37 Ressing ME et al. Occasional memory cytotoxic T cell response of patients with human papillomavirus type 16-positive cervical lesions against a human leucocyte antigen-A0201-restricted E7encoded epitope. Cancer Res 1996; 56: 582-588. 
38 Hollingsworth S, Gaken J, Darling D. Induction of tumour rejection by combination B7.1/IL-2 expressing tumour cells. Cancer Gene Ther 1995; 2: 240 (Abstr.).

39 Salvadori $\mathrm{S}$ et al. B7-1 amplifies the response to interleukin-2 secreting tumour vaccines in vivo, but fails to induce a response by nave cells in vitro. Hum Gene Ther 1995; 6: 1299-1306.

40 Wigler $\mathrm{M}$ et al. Transformation of mammalian cells with genes from prokaryotes and eukaryotes. Cell 1979; 16: 777-785.

41 Graham FL, Prevec L. Manipulation of adenovirus vectors. In: Murray EJ (ed). Methods of Molecular Biology, vol 7. Humana Press: Clifton, NJ, 1991, pp 109-128.

42 Wilkinson GW, Akrigg A. Constitutive and enhanced expression from the CMV major IE promoter in a defective adenovirus vector. Nucleic Acids Res 1992; 20: 2233-2239. 